\title{
Maintaining a clinical learning environment for medical students during a pandemic
}

\author{
Kirsty Morrison \\ Harrogate District Hospital, UK \\ Sally West \\ Harrogate District Hospital, UK \\ Kathryn Hogg \\ Harrogate District Hospital, UK
}

Keywords: medical education; HDFT; clinical skills; OSCE; clinical placement; Covid-19.

\section{The challenge}

We are a team of Ward-based Clinical Teachers based at a District General Hospital in the North of England. Our role as ward based clinical educators is to support medical students on their placements and to help them achieve their objectives, focussing mainly on thirdyear medical students, coaching them in clinical and practical skills. We offer hourly oneto-one sessions with guidance and feedback, sign off the students' ward-based assessments, and help them to prepare for their OSCE exam.

As a result of the pandemic, the challenge we faced was how to adapt the third-year placements to ensure that students received a comprehensive learning experience. We had to balance this with the demands of the hospital and changes in the configuration of services that occurred because of the trust's response to the pandemic. Each placement consists of 18 students equally divided between medicine, general surgery, and elderly care. The placements were five weeks long, starting in September 2021, with five placement rotations spaced over the academic year.

We identified four particular challenges that required addressing in order for us to deliver an effective placement. 
The first challenge was preventing the potential for overcrowding on the wards. The Health and Safety Executive's (2021) advice was that where social distancing cannot be maintained, the number of people should be kept to a minimum. There were already issues with too many students on a multi-speciality ward; this was worsened with the growing need for Covid-19 speciality wards and the cancelations of elective surgeries. This resulted in 12 students being based on the same ward, instead of being spread out over two wards and two theatres suites. The second challenge regarded student safety and mental health. The stressors created by epidemics is well documented (Son et al., 2020), and we were conscious of the effects for students coming into the clinical environment during the pandemic. Papapanou et al. (2021) have shown that students' mental health was of critical importance during this challenging period. Pre-pandemic, we had scheduled initial, mid-, and end of placement interviews with students; in view of this finding, we were determined that these would continue. The third challenge was the withdrawal of access to the student common room as social distancing could not be maintained there. This removed access to the noticeboard, therefore communication with the students was hampered. The fourth and final challenge was the issue of students self-isolating at home. We were conscious of the impact of this lost placement time and tried to find ways we could offer some support to isolating students.

\section{The response}

To overcome the overcrowding on the wards we formalised timetables and grouped the students into pairs. We contacted other speciality wards and departments to ask if they could accommodate students on an allocated time/day; as such we were able to place each pair into different clinical areas and thus reduce the number of students assigned to the ward each day. This provided broader exposure to the specialities, which was something that students had stated had been lacking pre-pandemic. To coincide with our interviews, we adapted our trust's health risk assessment, and asked the students to complete this on induction, discussing any concerns or health issues identified. To date we have been able to ensure that any placement related anxieties were addressed, and that plans have been implemented to help manage them. We were allocated an android mobile device which opened up the possibility of using communication platforms such as WhatsApp. We gained consent from the students to use their mobile numbers and created 
speciality specific WhatsApp groups. This proved a huge success as students were able to communicate with each other and the Ward-based Teacher team easily. They were also able to use this platform to secure individual sessions with us. We also used the free version of the Miro app, a virtual noticeboard, and shared placement information on there. Due to our roles being word-based we were limited in what we could offer the students who were isolating. We emailed web links that aided their self-study and ensured they were on Microsoft Teams for the group teaching sessions offered.

\section{Recommendations}

When we were first tasked with the challenge of adapting the medical students' placement, we were understandably overwhelmed. However, we learned that this could in fact be a catalyst for unforeseen opportunities, a realisation that continues to affect our work today. The timetables have enabled the students to feel part of the team and have ensured all aspects of their learning objectives have been addressed.

Ensuring we continue to provide our individual support to the students has been of paramount importance, particularly in these uncertain times, bearing in mind how overwhelmed students often feel on placement. We missed having access to the student common room, especially as this acted as a facilitating environment for discussion and reflection. The General Medical Council (GMC, 2019) state that 'taking time to reflect on experiences that have made you think or question your ideas or values is important for your individual wellbeing and development as a professional'; for this to occur in future, it is imperative that the common room is reintroduced. We found that the use of WhatsApp has helped with the dissemination of information and has also acted as a platform for the students to communicate with the rest of their placement group. This helped to bridge a gap in communication; we will be keeping this option for future groups in the hope that it can be enhanced with the use of the student common room as social distancing restrictions relax.

Ultimately, we found that there was no alternative we could provide to replace the clinical placement, despite sharing certain learning resources. We ensured that we spoke with students who had missed elements of their placement on their return and prioritised their 
learning objectives as much as possible. Papapanou et al. (2021) suggest that, despite best intentions, there are certain elements of the placement experience which cannot be successfully replaced by digital technology. Our role as clinical supervisors, for example, was inevitably constrained in ways that we could not fully overcome.

Overall, we have learnt that by embracing the challenge we faced, we have found a better way of providing a comprehensive placement for students. We took advantage of the camaraderie that was evident at the beginning, forging better relationships with other departments, and this has introduced more variety into the placements. This has been verified by the feedback the university has recently provided and it therefore supports us in maintaining these changes.

\section{References}

Health and Safety Executive (2021) Social distancing to make your workplace COVIDsecure. Available at: https://www.hse.gov.uk/coronavirus/social-distancing/unableto-social-distance.htm (Accessed: 23 June 2021).

General Medical Council (2019) The reflective practitioner - a guide for medical students. Available at: https://www.gmc-uk.org/education/standards-guidance-andcurricula/guidance/reflective-practice/the-reflective-practitioner---a-guide-formedical-students (Accessed: 23 June 2021).

Papapanou, M., Routsi, E., Tsamakis, K, Fotis, L., Marinos, G., Papaioannou, T., Tsiptsios, D., Smyrnis, N., Rizos, E. and Schizas, D. (2021) 'Medical education challenges and innovations during COVID-19 pandemic', Postgraduate Medical Journal. https://doi.org/10.1136/postgradmedj-2021-140032.

Son, C., Hedge, S., Smith, A., Wang, X. and Sasangohar, F. (2020) 'Effects of COVID-19 on college students' mental health in the United States: interview survey study', Journal of Medical Internet Research, 22(9). https://doi.org/10.2196/21279. 


\section{Author details}

Kirsty Morrison, Sally West, and Kathryn Hogg are Ward-based Clinical Teachers at Harrogate District Hospital and honorary tutors at Leeds Institute of Medical Education. They are registered nurses who educate, assess, and support Medical students from Leeds Institute of Medical Education, focusing mainly on the third-year students but they also help support the fourth- and fifth-years when required. 